\title{
Multiple endocrine neoplasia syndrome type 1: institution, management, and data analysis of a nationwide multicenter patient database
}

\author{
Francesca Giusti ${ }^{1}$ - Luisella Cianferotti ${ }^{1}$ - Francesca Boaretto ${ }^{2}$ - Filomena Cetani ${ }^{3}$ - Federica Cioppi ${ }^{1}$. \\ Annamaria Colao $^{4}$ - Maria Vittoria Davi ${ }^{5}$ Antongiulio Faggiano ${ }^{6}$ - Giuseppe Fanciulli ${ }^{7} \cdot$ Piero Ferolla $^{8}$. \\ Diego Ferone ${ }^{9} \cdot$ Caterina Fossi $^{1} \cdot$ Francesco Giudici $^{1} \cdot$ Giorgio Gronchi $^{1} \cdot$ Paola Loli $^{10} \cdot$ Franco Mantero $^{11}$. \\ Claudio Marcocci ${ }^{3}$ - Francesca Marini ${ }^{1}$ - Laura Masi $^{1}$ - Giuseppe Opocher ${ }^{2}$ - Paolo Beck-Peccoz ${ }^{12}$. \\ Luca Persani ${ }^{13,14}$ - Alfredo Scillitani ${ }^{15}$ - Giovanna Sciortino ${ }^{13,14}$ - Anna Spada ${ }^{16}$ - Paola Tomassetti ${ }^{17}$. \\ Francesco Tonelli ${ }^{1} \cdot$ Maria Luisa Brandi ${ }^{1}{ }^{1}$
}

Received: 12 September 2016 / Accepted: 13 January 2017 / Published online: 28 January 2017

(C) Springer Science+Business Media New York 2017

\begin{abstract}
Objective The aim of this study was to integrate European epidemiological data on patients with multiple endocrine neoplasia type 1 by creating an Italian registry of this syndrome, including clinical and genetic characteristics and therapeutic management.

Methods Clinical, familial and genetic data of patients with multiple endocrine neoplasia type 1, diagnosed, treated, and followed-up for a mean time of 11.3 years, in 14 Italian referral endocrinological centers, were collected, over a 3-
\end{abstract}

year course (2011-2013), to build a national electronic database.

Results The Italian multiple endocrine neoplasia type 1 database includes 475 patients ( 271 women and 204 men), of whom 383 patients $(80.6 \%)$ were classified as familial cases (from 136 different pedigrees), and 92 (19.4\%) patients were sporadic cases. A MEN1 mutation was identified in $92.6 \%$ of familial cases and in $48.9 \%$ of sporadic cases. Four hundred thirty-six patients were symptomatic, presenting primary hyperparathyroidism, gastroenteropancreatic neuroendocrine
Maria Luisa Brandi

marialuisa.brandi@unifi.it

1 Department of Surgery and Translational Medicine, University of Florence, Largo Palagi 1, 50139 Florence, Italy

2 Familial Tumor Unit, Veneto Institute of Oncology IOV-IRCCS, Padua, Italy

3 Department of Clinical and Experimental Medicine, Section of Endocrinology, University of Pisa, Pisa, Italy

4 Endocrinology Unit, Department of Clinical Medicine and Surgery, University of Naples Federico II, Naples, Italy

5 Internal Medicine, Section of Endocrinology, Department of Medicine, University of Verona, Verona, Italy

6 Thyroid and Parathyroid Surgery Unit, Istituto Nazionale per lo Studio e la Cura dei Tumori "Fondazione G. Pascale" IRCCS, Naples, Italy

7 NET Unit, Department of Clinical and Experimental Medicine, University of Sassari, Sassari, Italy

8 Department of Internal Medicine and Endocrine Sciences, University of Perugia, Perugia, Italy
$9 \quad$ Endocrinology Units, Department of Internal Medicine and Medical Specialties (DiMI), University of Genoa, Genoa, Italy

10 Department of Endocrinology, Hospital Niguarda Ca' Granda, Milan, Italy

11 Division of Endocrinology, Department of Medicine (DIMED), University of Padua, Padua, Italy

12 Professor Emeritus of the University of Milan, Milan, Italy

13 Department of Clinical Sciences and Community Health, University of Milan, Milan, Italy

14 Division of Endocrine and Metabolic Diseases, IRCCS Istituto Auxologico Italiano, Milan, Italy

15 Unit of Endocrinology 'Casa Sollievo della Sofferenza' Hospital, IRCCS, San Giovanni Rotondo, Foggia, Italy

16 Department of Clinical Sciences and Community Health, Fondazione IRCCS Ca' Granda-Ospedale Maggiore Policlinico, University of Milan, Milan, Italy

17 Department of Medical and Surgical Sciences, S. Orsola-Malpighi University Hospital, Bologna, Italy 
tumors and pituitary tumors in 93,53 , and $41 \%$ of cases, respectively. Thirty-nine subjects, belonging to affected pedigrees positive for a MEN1 mutation, were asymptomatic at clinical and biochemical screening. Age at diagnosis of multiple endocrine neoplasia type 1 probands was similar for both familial and simplex cases (mean age 47.2 \pm 15.3 years). In familial cases, diagnosis of multiple endocrine neoplasia type 1 in relatives of affected probands was made more than 10 years in advance (mean age at diagnosis $36.5 \pm$ 17.6 years).

Conclusions The analysis of Italian registry of multiple endocrine neoplasia type 1 patients revealed that clinical features of Italian multiple endocrine neoplasia type 1 patients are similar to those of other western countries, and confirmed that the genetic test allowed multiple endocrine neoplasia type 1 diagnosis 10 years earlier than biochemical or clinical diagnosis.

Keywords MEN1 - Italian registry $\cdot$ Pituitary adenomas • Gastroenteropancreatic tumors $\cdot$ Primary hyperparathyroidism $\cdot M E N 1$ genetic test

\section{Introduction}

Multiple endocrine neoplasia type 1 (MEN1) syndrome (MIM\#131100) is an inherited rare neuroendocrine syndrome, affecting primarily parathyroid glands, diffuse neuroendocrine tissues of the gastroenteropancreatic tract, and the anterior pituitary gland [1]. MEN1 syndrome may manifest with occurrence of varying combinations of more than 20 endocrine and non-endocrine tumors and lesions. The gene responsible, the MEN1 tumor suppressor gene, was identified in 1997 at 11q13.1 locus (OMIM gene/locus number 613733) [2], and consists of 10 exons encoding a protein of 610/615 amino acid called menin. Affected subjects are clinically diagnosed by the presence of tumors in at least two of the three main MEN1 affected organs: parathyroids, endocrine pancreas and anterior pituitary [3, 4], or by the occurrence of one of these three main tumors plus one of first degree relatives affected by the syndrome. MEN1 can be inherited from the affected relative as an autosomal dominant trait, with high penetrance, in a great majority of cases (over 90\%), or it can occur sporadically (less than $10 \%$ of cases) from a heterozygote MEN1 mutation developed at embryo level [5]. In some cases, familial and sporadic forms of MEN1 are difficult to distinguish. Indeed, the family history may seem negative because of failure to recognize the disorder in family members, the death of the MEN1-mutated parent before the manifestation of symptoms, or the late onset of the disease in the affected parent. The familial form is defined in an affected subject who has at least one first-degree relative with (1) at least two MEN1 main endocrine tumors, or (2) the involvement of only one MEN1 main organ in association with a MEN1 germline mutation. The sporadic form is characterized by only one affected subject within a family with no history of the disease.

To date, over 1500 germinal and somatic mutations of the MEN1 gene $[6,7]$ have been identified in familial and sporadic cases. Mutation analysis of the MENI gene allows the early identification of asymptomatic carriers, years before any MEN1-associated biochemical and/or instrumental abnormalities are detected $[6,8]$. Unfortunately, as reported by the great majority of worldwide epidemiology studies, a genotype-phenotype correlation, like in MEN2 syndromes, has not clearly been identified in MEN1 syndrome, strongly reducing the possibility to foresee exact future clinical manifestations of the disease by the specific gene mutation [6, 9-12]. Recently, two studies by the "Groupe d'étude des tumors endocrines (GTE)" reported a trend for intrafamilial correlation in disease expression and severity and in heritability of MEN1 tumors $[13,14]$. The first study reported a twofold higher risk of death in patients bearing a MENI mutation affecting menin domain interacting with the transcription factor JunD [13]. The second evidenced a positive intra-familial correlation and trait heritability only for three specific MEN1associated tumor types: pituitary adenomas, adrenal tumors, and thymic tumors [14]. However, this intrafamilial correlation was shown to decrease progressively when the degree of the genetic relationship was increased, even in presence of the same MEN1 mutation. This suggests that additional modifying genetic factors, and not only the specific MEN1 mutation, influence the development of different MEN1 clinical phenotypes.

Many organs at high risk of tumor development in MEN1 syndrome, such as the duodenum, pancreas, and lungs (bronchial carcinoids), are not suitable for preventive ablative surgery and, thus, prophylactic tumor prevention is not possible, even in presence of a positive genetic test. However, MEN1 genetic screening in subjects suspected to be affected by MEN1 and in relatives of a MEN1 mutation carrier is strongly recommended for the correct diagnosis of the disease, and the early identification of carriers, to program specific routine MEN1-associated tumor screening via biochemical analysis and imaging procedures (beginning in early childhood and continuing for the entire life), to direct early surgical and/or pharmacological intervention, and to guide surgery of parathyroid tumors and thymus removal at the same time as parathyroid surgery. Indeed, as confirmed by Ramundo et al., an early genetic diagnosis for hereditary NET syndromes results in a reduction of morbidity and mortality rate [15]. Conversely, genetic screening of the 
MEN1 gene in the general population is not recommended due to the rare prevalence of the disease. Thus, MEN1 patients sometimes remain undiagnosed for long periods, even after the manifestation of related clinical symptoms.

The collection of clinical, biochemical, and genetic characteristics of unselected patients with rare disorders, such as MEN1, in multicentre-nationwide registries or databases, is a useful approach to increase knowledge of epidemiological aspects of the disease and the natural course and prognosis of single manifestations of the syndrome [16-20].

The primary aim of this study was to determine the time between the diagnosis of MEN1 in probands and the subsequent MEN1 diagnosis in family members through the analysis of a national multicenter database of patients. The secondary aim was to describe clinical manifestations and disease course in Italian patients with MEN1 using currently available diagnostic techniques.

\section{Patients and methods}

The Italian Registry of Multiple Endocrine Neoplasia (RINEM), within the Italian Society of Endocrinology, was established in 1991. In 2011, the study group created the "Italian MEN1 Database", and started constructing an electronic database of Italian patients affected by this syndrome, after obtaining an informed consent for data collection for registry purposes. This national computed database includes family history, and clinical and genetic data of MEN1 patients, obtained from 14 major referral centers for endocrine inherited tumors and MEN syndromes. These centers are located in 12 different cities, in 9 Italian regions, and represent the three main geographical areas of Italy (North, Center, South), serving patients from all 20 Italian Regions. The study was initially approved by the Internal Review Board of the University of Florence (coordinating center), and then by local ethic committees of participating centers. The study was also fully endorsed by the Italian Society of MEN1 patients ("Associazione Italiana Neoplasie Endocrine Multiple tipo 1 e 2").

Italian patients with a diagnosis of MEN1 were both retrospectively and prospectively entered in the database in the years 2011-2013, detailing family history, and clinical and genetic characteristics. All data were anonymous through the use of identification numbers. Duplicate records were avoided by comparing date of birth, initials, and specific MEN1 mutation, when available. Duplicate registrations were excluded for the data analysis. Currently, the registry includes of the patients who have been followed for more than 20 years, as well as patients followed since being added to the database. The mean time of the follow-up period is about 11.3 years.
Birth date, gender, clinical family history, first MEN1manifestation (type and age of onset), age of MEN1 genetic and/or clinical diagnosis, all MEN1-associated endocrine and non-endocrine lesions, and past and current medical and surgical treatments were recorded.

In agreement with international MEN1 guidelines [3], the diagnosis of MEN1 was established according to one of the three following criteria in all expert endocrine centers: (1) the occurrence of two or more of the main MEN1associated endocrine tumors (i.e., parathyroid adenoma, gastroenteropancreatic tumor, and pituitary adenoma), (2) the occurrence of one of the main MEN1-associated tumors and a first degree relative with a clinical diagnosis of MEN1, or (3), the identification of a germline MEN1 mutation (even in asymptomatic disease-free individuals who have not yet developed biochemical or radiological abnormalities indicative of MEN1 tumor development) [21]. In this way, patients included in our database are representative of the general Italian MEN1 population and they do not represent a specific patient group.

MEN1 cases were classified as familial when at least two MEN1 cases were clinically discovered or suspected within a pedigree or when a MEN1 mutation was identified in at least two family members.

For patients enrolled collected more than 20 years ago and retrospectively entered in the database, the clinical diagnosis of MEN1 was initially based on the presence of clinical symptoms and/or the instrumental detection of the presence of MEN1-associated pathological lesions. In the last two decades, the introduction of increasingly sensitive and specific biochemical screenings has favored the target identification (even 10 years before instrumental detection) of secreting tumors. Moreover, after the discovery of the MEN1 gene and the application of the syndrome-related genetic test, many patients (mostly within a pedigree with a known mutation) have been diagnosed early with MEN1, even before the onset of the disease and the manifestation of biochemical signs, or they have been diagnosed during childhood or adolescence, even if only in presence of a single MEN1 manifestation. The first clinical manifestation was defined as the first MEN1-associated lesion that was discovered. The age of first clinical manifestation was also registered. The age of MEN1 diagnosis was assumed as the age at which the syndrome was recognized definitively.

PHPT (primary hyperparathyroidism) was defined as symptomatic in presence of a history of kidney stones and/ or presence of osteoporosis; conversely, asymptomatic PHPT was diagnosed by routine biochemical tests, and patients did not present a clinical manifestation associated with persistent elevated serum PTH (parathyroid hormone) and/or calcium level.

GEP-NETs (gastroenteropancreatic neuroendocrine tumors) were defined non-functioning tumors if they were 
not secreting hormones or releasing only neuroendocrine polypeptides that are not responsible for a specific syndrome (i.e., pancreatic polypeptide, PP). Functioning tumors are defined if producing an excess of active hormones, such as gastric, insulin, vasoactive intestinal polypeptide (VIP), glucagon, and somatostatin and, thus, resulting in specific clinical syndromes.

The classic manifestations are Zollinger Ellison syndrome, hypoglycemia, watery diarrhea, and symptoms of hyperglycemia. Pituitary tumors were defined non-functioning tumors if they were not secreting hormones. They were defined as functioning tumors if producing an excess of active hormones, such as prolactin, growth hormone, adrenocorticotrophic hormone and follicle-stimulating hormone, luteinizing hormone, and thyroid-stimulating hormone. The classic manifestations are hyperprolactinemia (e.g., amenorrhea, infertility, and galactorrhea in women and impotence and infertility in men), acromegaly, and Cushing's disease. Furthermore, these tumors can compress adjacent structures such as the optic chiasm or normal pituitary tissue, causing visual disturbances and/or hypopituitarism.

Specific biochemical screenings, such as plasma calcium, PTH, prolactin and insulin-like growth factor 1, GH (growth hormone), ACTH (adrenocorticotropic hormone), luteinizing hormone, follicle-stimulating hormone, thyroidstimulating hormone, fasting serum gastrin, fasting plasma insulin and glucose, and glucagon, VIP, $\mathrm{PP}$, and $\mathrm{CgA}$, were performed every 1-2 years.

Mutational analysis of the MEN1 gene was undertaken in all MEN1 probands. Mutation screening was performed on genomic DNA, extracted by peripheral blood in EDTA, analyzing the coding region (exon 2-10) and the exonintron junctions (splicing sites) of the MEN1 gene via PCRbased sequencing. Obtained sequences were compared to wild type reference sequence of the MENI gene, and mutations were classified using the standard nomenclature for the description of human DNA sequence variants. When a MEN1 mutation was detected, the mutation screening was extended to first degree relatives of the proband, independently of the presence of specific MEN1-related signs and symptoms.

Data included in the database are presented as percentage, median with the relative standard deviations and range.

\section{Results}

Five hundred twenty-three patients were originally entered in the database (Fig. 1). Cases presenting a duplicate registration or not meeting the correct MEN1 diagnostic criteria were then excluded, including all subjects from MEN1 families with a negative MEN1 genetic test.
Subsequently, only 475 patients were included in the final analysis: 271 (57\%) females and 204 (43\%) males. Within the observation period, 20 patients $(4.2 \%)$ died because of MEN1-related causes.

In particular, 14 died due to malignant gastrinomas from the complications of uncontrolled acid peptic disease (4 intestinal perforations and 3 gastric hemorrhages) and from multiple liver metastases (7 liver failures), 4 for kidney failure (due to a non-recognized hyperparathyroidism) and 2 for cardiac complications due to electrolyte imbalance (cardiac arrest from ventricular fibrillation with hypokalemia). All deceased patients showed the association of at least two manifestations of the syndrome at the time of MEN1 diagnosis (late diagnosis).

The average age at death was 61.3 years \pm 10.7 (range 41-76). The average age at the first clinical manifestation was $41.55 \pm 14.2$ years (range 24-66), and the average age at diagnosis of MEN1 was $55.1 \pm 13.3$ years (range 25-76). MEN1 was diagnosed between 1982-2001. The deaths occurred between 1989-2004. Unfortunately, we have no further information on these deaths.

The analysis of family histories allowed us to identify $383(80.6 \%)$ familial cases belonging to 136 different pedigrees, and $92(19.4 \%)$ sporadic cases.

A MEN1 mutation was identified in 355 (92.6\%) familial cases and $45(48.9 \%)$ sporadic cases. Conversely, no mutations in the coding region and splicing sites of the MEN1 gene were detected in 75 patients $(15.8 \%$ of the whole sample). Within this group of patients negative for MEN1 mutations, 28 were familial cases belonging to 9 families, and 47 were sporadic cases.

In this series of patients, the mean age at diagnosis of MEN1 was $41.6 \pm 17.4$ years (range 2-82). Mean age at diagnosis of probands (both familial and sporadic cases) was $47.2 \pm 15.3$ years (range 11-82), higher than that of family members identified by the screening [mean age of $36.5 \pm 17.6$ years (range 2-80)]. Thirty-nine relatives included in the database remained disease-free for the duration of the study, namely, no abnormalities in biochemical tests or evidence of disease by instrumental examinations were detected. These latter subjects, diagnosed only by MEN1 genetic testing, had a mean age of $18.7 \pm 15.5$ years (range 1-75), and were excluded from further analysis of the database. The mean age at the first clinical manifestation was $37.2 \pm 11.8$ years (range 8-80). According to the MEN1 syndrome hallmark, PHPT, GEPNETs, and pituitary tumors were the three most prevalent lesions in our series: $93 \%$ (405 cases), 53\% (230 cases), and $41 \%$ (178 cases), respectively (Fig. 2).

The most frequent clinical phenotype resulted to be the combination PHPT/GEP-NETs (114 cases; 24\%), followed by PHPT/GEP-NETs/pituitary tumors (92 cases; 19.4\%) and pituitary tumors/GEP-NETs (8 cases; 1.7\%) (Fig. 3). 


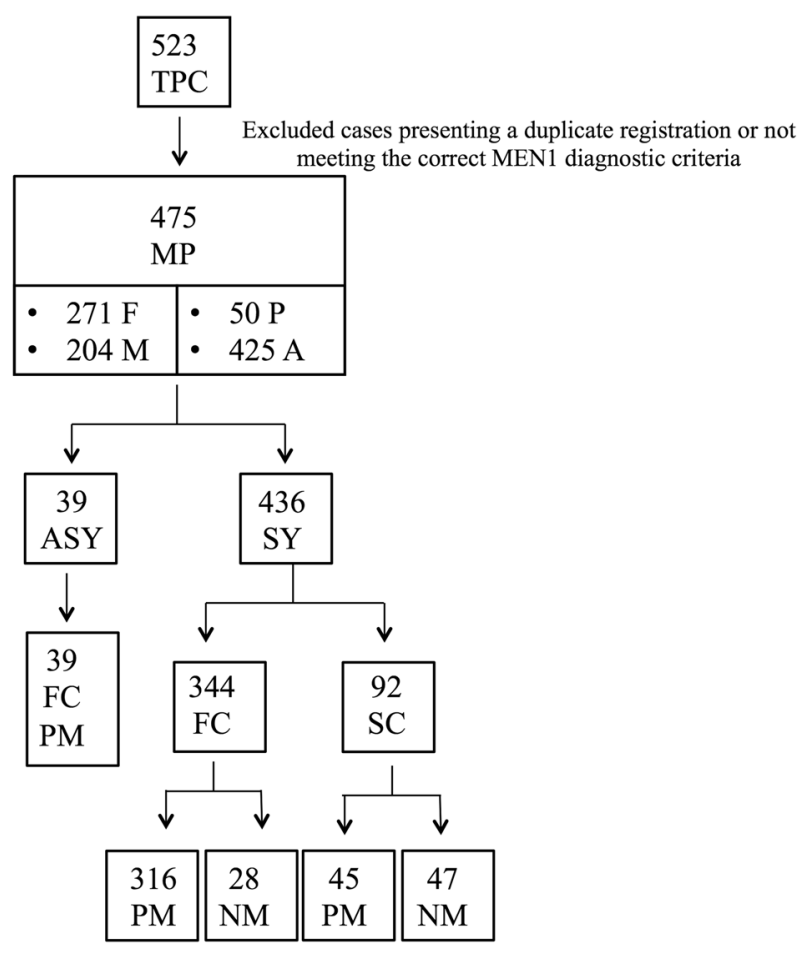

Fig. 1 Flow diagram of the study of the Italian MEN1 database. TPC: total patients enrolled; M: males; F: females; $P$ : pediatric (diagnosis before 18 years of age); A: adults; MP: MEN1 patients; ASY: asymptomatic carriers; SY: symptomatic patients; FC: familial carriers; SC: sporadic carriers; PM: positive MEN1 mutation; NM: negative MEN1 mutation

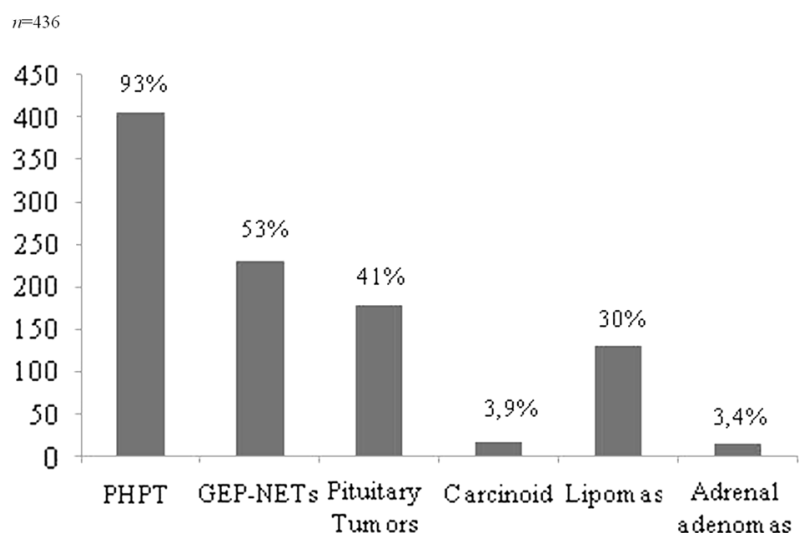

Fig. 2 Schematic representation of distribution of main MEN1-related lesions; $n=$ number of symptomatic subjects

In the following sections, we describe the clinical features in symptomatic patients with overt MEN1, mainly focusing on the mode of disease presentation.

\section{PHPT}

Four hundred and five patients [259/405 (64\%) females and 146/405 (36\%) males] presented with PHPT.

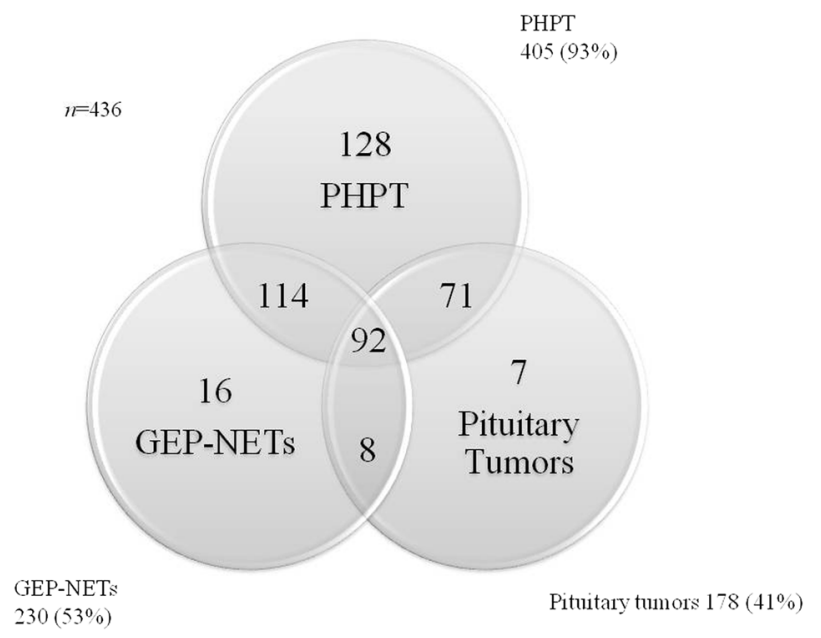

Fig. 3 Prevalence of PHPT, GEP-NETs and pituitary tumors; numbers of patients with two or three MEN1-related main features are shown within circle intersections; $n=$ number of symptomatic subjects

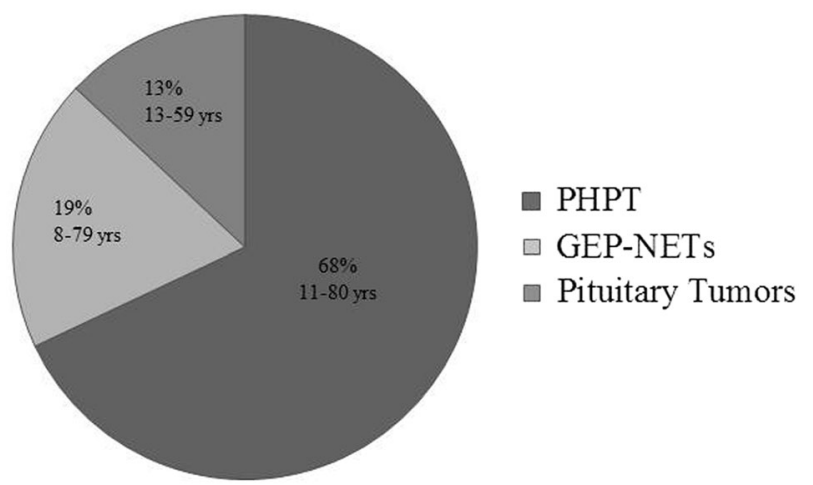

Fig. 4 Schematic representation of first diagnosed MEN1 tumor and diagnosis age in patients included in the Italian database

In 128 cases, PHPT was the only manifestation of MEN1 (29.4\% of all affected patients and $31.6 \%$ of patients presenting PHPT), while it was associated with other lesions in 277 cases $(63.5 \%$ of all affected patients and $68.4 \%$ of patients presenting PHPT). In particular, 92 cases showed PHPT associated with GEP-NETs and pituitary tumors, and 114 and 71 patients displayed PHPT associated with GEPNETs or pituitary tumors, respectively.

PHPT was the first MEN1 manifestation in 291 cases (67\% of all patients with overt MEN1 and $71.85 \%$ of patients with MEN1-related PHPT) (Fig. 4). The mean age at diagnosis of PHPT was $38.6 \pm 14.9$ years (range 11-80), while the mean age at which the diagnosis of MEN1 was made was $45.1 \pm 18.0$ (range 11-80). Of these 291, 141 (48.5\%) patients were symptomatic for PHPT and 150 (51.5\%) asymptomatic. Among the 291 cases in which PHPT was the first manifestation of MEN1, 145 (49.8\%) 
were probands (familial and sporadic cases) and 146 $(50.2 \%)$ were family members. In the 145 probands, the mean age at PHPT diagnosis was $43.8 \pm 14.0$ years (range 18-80) and the mean age at MEN1 diagnosis was $55.7 \pm$ 13.1 (range 19-80); of them, 131/145 (90.3\%) were symptomatic and $14 / 145(9.7 \%)$ were asymptomatic at the time of diagnosis. In the 146 family members, the mean age at PHPT diagnosis was $36.5 \pm 14.80$ years (range $11-80$ ); age at MEN1 diagnosis was 38.1 \pm 18.0 (range 11-80); of them 10/146 (6.8\%) were symptomatic and 136/146 $(93.2 \%)$ were asymptomatic at the time of diagnosis.

Information about PHPT characteristics, surgery and pharmacological treatment are summarized in Table 1.

\section{GEP-NETs}

Two hundred and thirty patients [117/230 (51\%) females and 113/230 (49\%) males] presented with GEP-NETs. In 16 cases GEP-NETs were the only manifestation of MEN1 (3.7\% of all affected patients and $7 \%$ of patients presenting GEP-NETs), while they were associated with other lesions in 214 cases $(49.08 \%$ of all affected patients and $93 \%$ of patients presenting GEP-NETs). In particular, 92 cases of GEP-NETs were associated with PHPT and pituitary tumors, and 114 and 8 patients displayed GEP-NETs associated with PHPT or pituitary tumors, respectively.

Non-functioning tumors were 136/230 (59\%) and functioning tumors were 94/230 (41\%). Among functioning tumors, gastrinoma was the most common with $61(26.6 \%$ of total GEP-NETs) cases, followed by $26(11.4 \%)$ insulinomas, $4(1.7 \%)$ glucagonomas, and $3(1.3 \%)$ vipomas.

GEP-NETs were the first MEN1 manifestation in 81 cases (18.6\% of all patients with overt MEN1 and 35.2\% of patients with MEN1-related GEP-NET) (Fig. 4). The mean age at diagnosis of GEP-s was $37.3 \pm 14.5$ years (range
8-79), while the mean age at which the diagnosis of MEN1 was made was $44.6 \pm 16.1$ (range 16-82). Within this group of 81 patients: (1) 14 (17.3\%) were non-functioning tumors and $67(82.7 \%)$ were functioning tumors; among functioning tumors, gastrinoma was the most common with 46 $(56.8 \%)$ cases, followed by $18(22.2 \%)$ insulinomas, 2 (2.4\%) vipomas, and one (1.2\%) glucagonoma; (2) the majority of patients had multiple tumors; clinically, all functioning tumors occurred with the classic manifestations: Zollinger Ellison syndrome, hypoglycemia, watery diarrhea or symptoms of hyperglycemia.

Of these 81 cases, $50(61 \%)$ were probands (familial and sporadic cases) and 31 (39\%) were family members. In the 50 probands, the mean age at GEP-NETs diagnosis was $35.8 \pm 14$ years (range 14-59) and the mean age at MEN1 diagnosis was $49.3 \pm 15.3$ (range 17-75); 42/50 (84\%) patients had functioning tumors with classic clinical manifestations (e.g., Zollinger Ellison syndrome, hypoglycemia, watery diarrhea and symptoms of hyperglycemia); gastrinoma was the most common, consisting of $27(64 \%)$ cases, followed by 12 (24\%) insulinomas, 2 (4\%) vipomas, and 1 (2\%) glucagonoma); 8/50 (16\%) patients had nonfunctioning tumors diagnosed by instrumental investigation of the gastroenteropancreatic tract.

In the 31 family members, the mean age at GEP-NETs diagnosis was $38.4 \pm 15$ years (range 8-79) and the mean age at MEN1 diagnosis was 38.5 \pm 16.7 (range 25-82); $25 / 31(80.6 \%)$ patients had functioning tumors with classic clinical manifestations (e.g., Zollinger Ellison syndrome, hypoglycemia, watery diarrhea, and symptoms hyperglycemic); gastrinoma was the most common, consisting of $19(76 \%)$ cases, followed by 6 (24\%) insulinomas; 6/31 (19.4\%) had non-functioning tumors detected by instrumental investigation of the gastroenteropancreatic tract.

Table 1 Characteristics and management of PHPT

\begin{tabular}{|c|c|c|c|c|c|c|c|}
\hline \multirow[t]{2}{*}{ PHPT } & \multirow[t]{2}{*}{ Total $n(\%)$} & \multicolumn{2}{|c|}{ Clinical $n(\%)$} & \multicolumn{2}{|l|}{ Surgery $n(\%)$} & \multirow{2}{*}{$\begin{array}{l}\text { Pharmacological } \\
\text { approach } \\
\text { (calcimimetics) } n(\%)\end{array}$} & \multirow{2}{*}{$\begin{array}{l}\text { Follow-up } \\
n(\%)\end{array}$} \\
\hline & & $\mathrm{SY} n(\%)$ & $\operatorname{ASY} n(\%)$ & TPTX $n(\%)$ & SPTX or LSPTX $n(\%)$ & & \\
\hline \multirow[t]{2}{*}{ All patients } & $405(93 \%)$ & $159(39 \%)$ & $246(61 \%)$ & \multicolumn{2}{|l|}{$253(62.5 \%)$} & $68(16.8 \%)$ & $84(20.7 \%)$ \\
\hline & & & & $65(30 \%)$ & $151(70 \%)$ & & \\
\hline \multirow{4}{*}{$\begin{array}{l}\text { First manifestation } \\
\text { - Total patients } \\
\text { - Probands }\end{array}$} & $291(67 \%)$ & $141(48.5 \%)$ & $150(51.5 \%)$ & \multicolumn{2}{|l|}{$167(57.3 \%)$} & $40(13.7 \%)$ & $84(29 \%)$ \\
\hline & & & & $51(30.5 \%)$ & $116(69.5 \%)$ & & \\
\hline & $145(49.8 \%)$ & $131(90.3 \%)$ & $14(9.7 \%)$ & \multicolumn{2}{|l|}{$115(79.3 \%)$} & $10(6.9 \%)$ & $20(13.8 \%)$ \\
\hline & & & & $35(30.4 \%)$ & $80(69.6 \%)$ & & \\
\hline \multirow[t]{2}{*}{ - Family members } & $146(50.2 \%)$ & $10(6.8 \%)$ & $136(93.2 \%)$ & \multicolumn{2}{|l|}{$52(35.6 \%)$} & $30(20.5 \%)$ & $64(43.9 \%)$ \\
\hline & & & & $16(30.7 \%)$ & $36(20.5 \%)$ & & \\
\hline
\end{tabular}

TPTX total parathyroidectomy, SPTX subtotal parathyroidectomy, LSPTX less than subtotal parathyroidectomy, $A S Y$ asymptomatic carriers, $S Y$ symptomatic patients 
Information about GEP-NET characteristics, surgery, and pharmacological treatment are summarized in Table 2.

\section{Pituitary tumors}

One hundred and seventy-eight patients [105/178 (59\%) females and 73/178 (41\%) males] presented with pituitary tumors. In 7 cases, pituitary tumors were the only manifestation of MEN1 (1.6\% of all affected patients and 3.9\% of patients presenting pituitary tumors), while they were associated with other lesions in 173 patients $(39.7 \%$ of all affected patients and $97.2 \%$ of patients presenting pituitary tumors). In particular, 92 cases showed pituitary tumors associated with PHPT and GEP-NETs, and 71 and 8 patients displayed pituitary tumors associated with PHPT or GEP-NETs, respectively.

One hundred and twelve of $178(63 \%)$ pituitary tumors were microadenomas. Non-functioning tumors were $36 / 178$ (20.2\%) and functioning tumors were 142/178 (79.8\%). Among functioning tumors, prolactinoma was the most common with 120 (67.4\% of total) cases, followed by 12 (6.7\%) GHomas, 8 (4.4\%) ACTHomas, 1 (0.6\%) prolactin-ACTH adenoma and 1 prolactin-GH adenoma $0.6 \%$ (1/178).

Pituitary tumors were the first manifestation in 56 cases (12.8\% of all patients with overt MEN1 and $31.4 \%$ of patients with MEN1-related pituitary adenomas) (Fig. 4). The mean age at diagnosis was $33.4 \pm 14.7$ years (range 13-59), while the mean age at MEN1 diagnosis was $38.7 \pm$ 15.7 (range 7-73).

Within this group of 56 patients: $3(5.4 \%)$ were nonfunctioning tumors, and $53(94.65 \%)$ were functioning tumors; among functioning tumors, prolactinoma was the most common with 43 (76.8\%) cases, followed by 4 (7.1\%) GHomas, 4 (7.1\%) ACTHomas, one (1.8\%) PL-ACTH adenoma and one (1.8\%) PL-GH adenoma; clinically, pituitary adenomas occurred with symptoms related to acromegaly in $2(3.6 \%)$ cases, Cushing's syndrome in 2 (3.6\%) cases, headache in $10(17.8 \%)$ cases, galactorrhea in 6 $(10.7 \%)$ cases, oligomenorrhea in $10(17.8 \%)$ cases, and hypogonadism in $8(14.3 \%)$ cases; $18 / 56(32.1 \%)$ cases were diagnosed during biochemical and/or instrumental screening.

Of these 56 cases, 26 (46.4\%) were probands (familial and sporadic cases) and $30(53.6 \%)$ were family members. In the 26 probands, the mean age at pituitary tumor diagnosis was $31.7 \pm 14.8$ years (range 17-65) and the mean age at MEN1 diagnosis was 39.8 \pm 14.1 (range 14-65); all 26 cases were functioning tumors, with prolactinoma being the most common, consisting of 19 (73.1\%) cases, followed by 2 (7.7\%) GHomas, 4 (15.4\%) ACTHomas, and 1 (3.8\%) PL-ACTH adenoma.

In the 30 family members, the mean age at pituitary tumor diagnosis was $33.6 \pm 14.9$ years (range 7-73) and the mean age at MEN1 diagnosis was 37.6 \pm 16.7 (range 7-73);

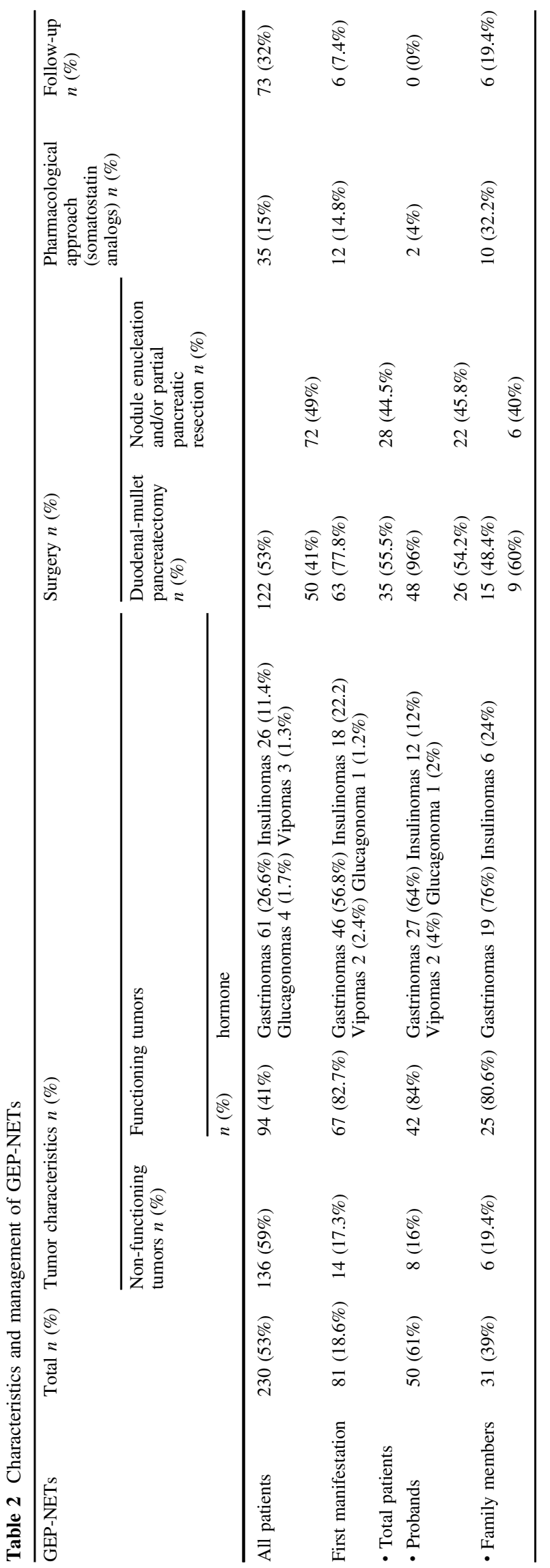


$3(10 \%)$ were non-functioning tumors and $27(90 \%)$ were functioning tumors, with prolactinoma being the most common, consisting of $24(80 \%)$ cases, followed by 2 (6.6\%) GHomas and 1 (3.3\%) PL-GH adenoma.

Information about pituitary adenoma characteristics, surgery, and pharmacological treatment are summarized in Table 3 .

\section{Thymic, bronchopulmonary, and gastric carcinoids}

Seventeen carcinoid tumors were detected (3.9\%) (Fig. 2); all of them were associated with other MEN1 lesions. Of these carcinoids, $11 / 17(64.8 \%)$ were located in bronchial tubes, $3 / 17(17.6 \%)$ in the thymus, and $3 / 17(17.6 \%)$ in the gastrointestinal tract (one in the ileum and two in the stomach). Thymic and bronchial carcinoids were diagnosed by radiological imaging (computed tomography and/or magnetic resonance imaging), which may be associated with Octreotide scintigraphy. Gastric carcinoids were mainly detected at the time of gastric endoscopy for GEP-NETs.

In females, carcinoids occurred predominantly in bronchial tubes ( 8 cases), followed by the gastrointestinal tract ( 2 cases). Conversely, in males, carcinoids occurred mainly in the thymus ( 3 cases), followed by bronchial tubes ( 3 cases) and the gastrointestinal tract (1 case).

Carcinoid tumors were the first manifestation in 4 patients (1\% of all affected patients and $23.5 \%$ of patients presenting carcinoids); the mean age at carcinoid diagnosis was $45.2 \pm 12$ years (range 32-66) and the mean age at MEN1 diagnosis was $47.7 \pm 10.9$ (range 30-71).

Within these 4 cases: bronchopulmonary carcinoids were the most common with $3(75 \%)$ cases (3/4), followed by $1(25 \%)$ carcinoid of the gastrointestinal tract. At the time of diagnosis all patients had clinical features of the carcinoid syndrome.

\section{Lipomas}

Lipomas occurred in 130 (30\%) (Fig. 2) patients with MEN1. They were multiple and subcutaneous in $117 / 130$ $(90 \%)$ patients and visceral in 13/130 (10\%) cases.

In one $(0.25 \%)$ patient, lipomas were the first clinical manifestation of the disease. Age at lipoma diagnosis was 45 years, while age at MEN1 diagnosis was 52 years.

Clinical and therapeutic management was conservative in $91 / 130(70 \%)$ patients.

\section{Adrenal adenomas}

Adrenal adenomas occurred in 15 (3.4\%) (Fig. 2) patients with MEN1. In 13/15 (86\%) cases, they were asymptomatic adrenocortical tumors.

Adrenal adenomas were the first clinical manifestation in $2(0.5 \%$ of all affected patients and $13.3 \%$ of patients presenting adrenal adenomas) patients; the mean age at adrenal adenoma diagnosis was $40 \pm 4$ years (range 36-44) and the mean age at MEN1 diagnosis was $42 \pm 4$ (range 38-46).

\section{Discussion}

National or supranational databases and registries are of pivotal importance for clinical and translational research and knowledge in the field of rare diseases [22]. MEN1 is a rare endocrine disease and, because of its complexity, patients are usually referred to highly specialized tertiary centers. The present study is the result of the efforts of a study group within the Italian Society of Endocrinology and describes MEN1 in the Mediterranean country of Italy.

The results of this study refer to patients with a long observation period, followed-up in multicenter endocrinological referral centers for this disease, applying the same international guidelines for MEN1 diagnosis and management.

MEN1 is a congenital syndrome, with a high degree of penetrance within the fifth decade of life [21]. Due to the autosomal dominant pattern of inheritance, the disease presents an equal incidence in both genders [20]. However, in the Italian patient series, a greater predominance (57 vs. $43 \%$ ) of female patients was detected, confirming what has been previously shown in similar studies carried out in France, the Netherlands and Japan [17-20, 23, 24].

Morbidity of the syndrome is caused principally by abnormal hormone secretion from MEN1-associated neuroendocrine tumors. Mortality is principally due to malignant gastrinomas and carcinoids. In the last two decades, the rate of complications associated with MEN1 has declined thanks to early diagnosis and improvement of therapeutic strategies [25, 26]. Biochemical screening for functioning tumors allows their detection 10 years earlier than clinical manifestations and/or imaging studies, providing the possibility of early intervention [27]. Our series appears to show that malignant gastrinomas were the most frequent MEN1-related causes of death, despite the effectiveness of long-term medical management or surgical therapy. In these cases, the disease was indeed diagnosed in an advanced stage, and it explains why the results of this study are in apparent contrast to more recent series taking into account patients diagnosed in earlier stages [28].

Since the identification of the MEN1 gene in 1997, the genetic test has made earlier diagnosis possible, allowing clinical surveillance and screenings in mutation carriers well before tumor occurrence and/or progression. Data from the Italian database confirm that, thanks to the genetic test, the average age of MEN1 diagnosis is about 10 years younger in family members as compared with that of the proband itself. 


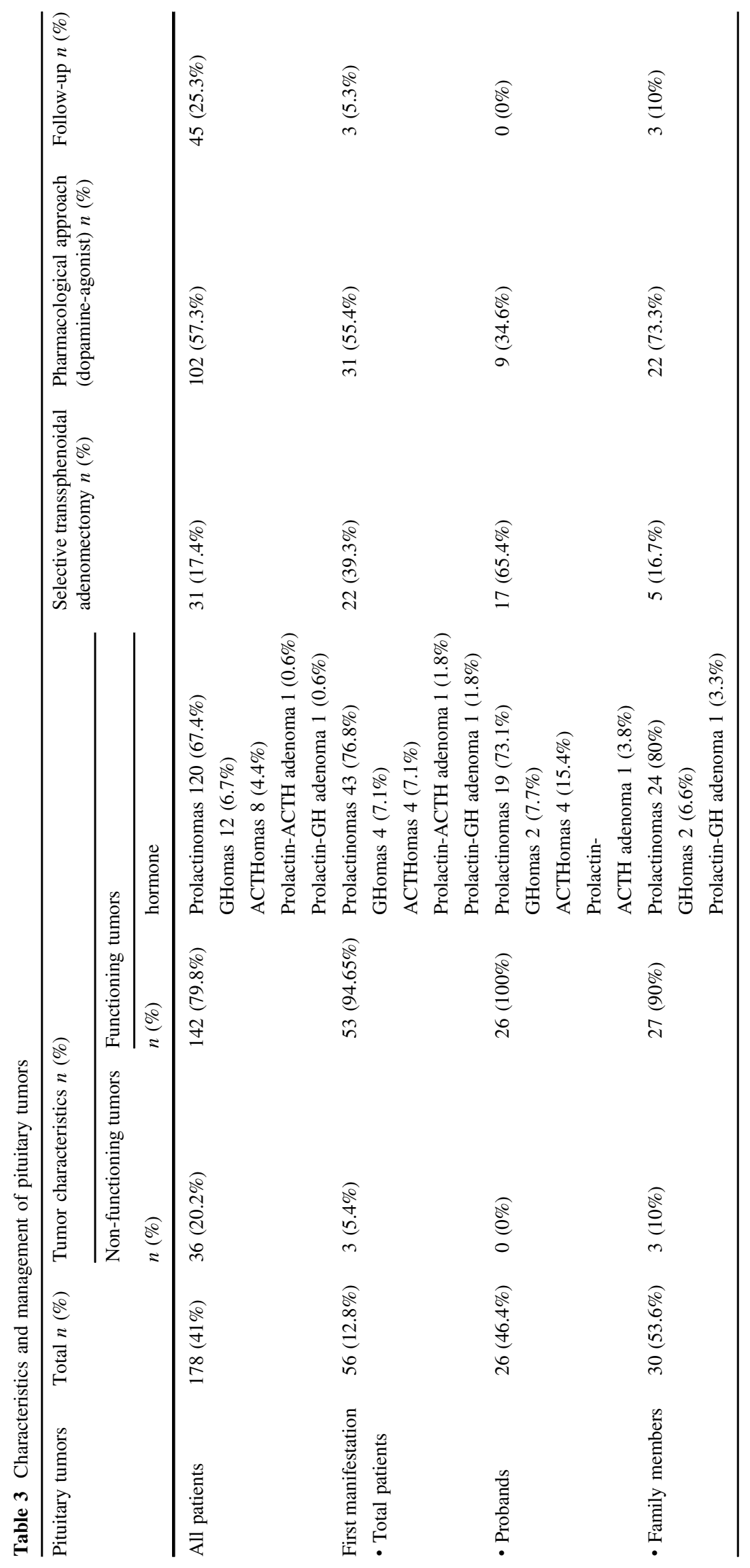


This is at variance with data obtained in other European countries, where a delay in diagnosis in family members has been reported [29]. Moreover, the mean average age of the first clinical manifestation in probands occurred about 10 years before the diagnosis of MEN1, whereas in familial members the average age of MEN1 diagnosis occurred about 1 year before the first clinical manifestation of the syndrome. The great majority of the asymptomatic diseasefree patients has been diagnosed by genetic testing after the identification of their pedigree MEN1 mutation.

Our data confirm that, as in other series, PHPT, GEPNETs and pituitary tumors are the three most prevalent lesions in MEN1. PHPT was the most frequent clinical feature (93\% of cases) and the most frequent first clinical manifestation (67\% of cases) of MEN1 [21].

The diagnosis of the most prevalent MEN1-associated tumors (parathyroid tumors, GEP-NETs and anterior pituitary adenomas) was performed earlier (38. 6 years) than in patients with their sporadic counterparts in the general population (55 years) [21]. The mean age at diagnosis of MEN1-related gastrinoma was 41 years, about 1.5-2 decades earlier than in non-syndromic sporadic gastrinoma. Similarly, the mean age at diagnosis (21.5 years) of MEN1related insulinoma was about two decades earlier than in the sporadic form $[3,21]$.

The mutation analysis performed in all MEN1 probands identified a germinal heterozygote inactivating mutation of the MEN1 gene in $92.7 \%$ of familial cases and in $48.9 \%$ of sporadic cases. No MEN1 mutation was identified in $15.8 \%$ of our MEN1 patients. The percentage of negative genetic tests is consistent with data previously reported in literature [6]; these patients could bear a large gene deletion or insertion of non-detectable by Sanger's sequencing analysis, could have a mutation in a region of MENI gene noncommonly analyzed by the analysis (introns or unknown regulatory regions), or could be phenocopies (i.e., MEN4 syndrome, which is indeed caused by mutations in the $C D N K 1 B$ gene).

Genetic screening allows the early identification of asymptomatic carriers of mutations among family members and grants the possibility to direct them to a specific program of biochemical and imaging surveillance, according to the international guidelines for MEN1 syndrome [3, 21]. Currently, 39 asymptomatic MENI carriers (mean age 18.7 years) who have not yet shown clinical and biochemical manifestations of the syndrome are under clinical surveillance according to international guidelines [3, 21].

Unfortunately, as in other cases [23, 24], our database presents some limitations. First, for patients included retrospectively, the family and clinical information may be incomplete. Second, the relatively short follow-up period may not provide long-term information about clinical manifestations and disease complications. Nonetheless, the institution of the dynamic database will allow the insertion of new entries and the possibility to add information during the course of the disease, to gather more data on the disease and, hopefully, to establish possible correlations between the clinical phenotype and MEN1 mutations.

Acknowledgements This study was supported by one unrestricted grant from Fondazione Ente Cassa di Risparmio di Firenze to ML Brandi, and by AIMEN $1 \& 2$. There is no funding associated with this publication.

\section{Compliance with Ethical Standards}

Conflict of Interest The authors declare that they have no competing interest.

Ethical Approval All procedures performed in studies involving human participants were in accordance with the ethical standards of the institutional and/or national research committee and with the 1964 Helsinki declaration and its later amendments or comparable ethical standards.

Informed consent Informed consent was obtained from all individual participants included in the study.

\section{References}

1. A. Falchetti, F. Marini, E. Luzi, F. Giusti, L. Cavalli, T. Cavalli, M.L. Brandi, Multiple endocrine neoplasia type 1 (MEN1): not only inherited endocrine tumors. Genet. Med. 11(12), 825-835 (2009)

2. S.C. Chandrasekharappa, S.C. Guru, P. Manickam, S.E. Olufemi, F.S. Collins, M.R. Emmert-Buck, L.V. Debelenko, Z. Zhuang, I. A. Lubensky, L.A. Liotta, J.S. Crabtree, Y. Wang, B.A, Roe, J. Weisemann, M.S. Boguski, S.K. Agarwal, M.B. Kester, Y.S. Kim, C. Heppner, Q. Dong, A.M. Spiegel, A.L. Burns, S.J. Marx, Positional cloning of the gene for multiple endocrine neoplasiatype 1. Science 276(5311), 404-407 (1997)

3. M.L. Brandi, R.F. Gagel, A. Angeli, J.P. Bilezikian, P. BeckPeccoz, C. Bordi, B. Conte-Devolx, A. Falchetti, R.G. Gheri, A. Libroia, C.J. Lips, G. Lombardi, M. Mannelli, F. Pacini, B.A. Ponder, F. Raue, B. Skogseid, G. Tamburrano, R.V. Thakker, N. W. Thompson, P. Tomassetti, F. Tonelli, S.A. Wells Jr., S.J. Marx, Guidelines for diagnosis and therapy of MEN type 1 and type 2. J. Clin. Endocrinol. Metab. 86(12), 5658-5671 (2001)

4. V. Fendrich, P. Langer, J. Waldmann, D.K. Bartsch, M. Rothmund, Management of sporadic and multiple endocrine neoplasia type 1 gastrinomas. Br. J. Surg. 94(11), 1331-1341 (2007)

5. F. Marini, S. Carbonell Sala, A. Falchetti, D. Caramelli, M.L. Brandi, The genetic ascertainment of multiple endocrine neoplasia type 1 syndrome by ancient DNA analysis. J. Endocrinol. Invest. 31(10), 905-909 (2008)

6. M.C. Lemos, R.V. Thakker, Multiple endocrine neoplasia type 1 (MEN1): analysis of 1336 mutations reported in the first decade following identification of the gene. Hum. Mutat. 29(1), 22-32 (2008)

7. P. Concolino, A. Costella, E. Capoluongo, Multiple endocrine neoplasia type 1 (MEN1): An update of 208 new germline variants reported in the last 9 years. Cancer Genet. 209(1-2), 36-41 (2016) 
8. J. Waldmann, V. Fendrich, N. Habbe, D.K. Bartsch, E.P. Slater, P. H. Kann, M. Rothmund, P. Langer, Screening of patients with multiple endocrine neoplasia type 1 (MEN-1): a critical analysis of its value. World J. Surg. 33(6), 1208-1218 (2009)

9. M.A. Kouvaraki, J.E. Lee, S.E. Shapiro, R.F. Gagel, S.I. Sherman, R.V. Sellin, G.J. Cote, D.B. Evans, Genotype-phenotype analysis in multiple endocrine neoplasia type 1. Arch. Surg. 137(6), 641-647 (2002)

10. J.J. Turner, P.D. Leotlela, A.A. Pannett, S.A. Forbes, J.H. Bassett, B. Harding, P.T. Christie, D. Bowen-Jones, S. Ellard, A. Hattersley, C.E. Jackson, R. Pope, O.W. Quarrell, R. Trembath, R.V. Thakker, Frequent occurrence of an intron 4 mutation in multiple endocrine neoplasia type 1. J. Clin. Endocrinol. Metab. 87, 2688-2693 (2002)

11. V. Wautot, C. Vercherat, J. Lespinasse, B. Chambe, G.M. Lenoir, C.X. Zhang, N. Porchet, M. Cordier, C. Beroud, A. Calender, Germline mutation profile of MEN1 in multiple endocrine neoplasia type 1: search for correlation between phenotype and the functional domains of the MEN1 protein. Hum. Mutat. 20, 35-47 (2002)

12. C.J. Lips, K.M. DreijerinkI, J.W. Höppener, Variable clinical expression in patients with a germline MEN1 disease gene mutation: clues to a genotype-phenotype correlation. Clinics 67 (1), 49-56 (2012)

13. J. Thevenon, A. Bourredjem, L. Faivre, C. Cardot-Bauters, A. Calender, A. Murat, S. Giraud, P. Niccoli, M.F. Odou, F. BorsonChazot et al. Higher risk of death among MEN1 patients with mutations in the JunD interacting domain: a Groupe d'etude des Tumeurs Endocrines (GTE) cohort study. Hum. Mol. Genet. 22 (10), 1940-1948 (2013)

14. J. Thevenon, A. Bourredjem, L. Faivre, C. Cardot-Bauters, A. Calender, M. Le Bras, S. Giraud, P. Niccoli, M.F. Odou, F. Borson-Chazot et al. Unraveling the intrafamilial correlations and heritability of tumor types in MEN1: a Groupe d'étude des Tumeurs Endocrines study. Eur. J. Endocrinol. 173(6), 819-826 (2015)

15. V. Ramundo, F. Milone, R. Severino, S. Savastano, C. Di Somma, L. Vuolo, L. De Luca, G. Lombardi, A. Colao, A. Faggiano, Clinical and prognostic implications of the genetic diagnosis of hereditary NET syndromes in asymptomatic patients. Horm. Metab. Res. 43(11), 794-780 (2011)

16. B. Gatta-Cherifi, O. Chabre, A. Murat, P. Niccoli, C. CardotBauters, V. Rohmer, J. Young, B. Delemer, H. Du Boullay, M.F. Verger, J.M. Kuhn, J.L. Sadoul, P. Ruszniewski, A. Beckers, M. Monsaingeon, E. Baudin, P. Goudet, A. Tabarin, Adrenal involvement in MEN1. Analysis of 715 cases from the Groupe d'etude des Tumeurs Endocrines database. Eur. J. Endocrinol. 166(2), 269-279 (2012)

17. D. Vezzosi, C. Cardot-Bauters, N. Bouscaren, M. Lebras, M. Bertholon-Grégoire, P. Niccoli, N. Levy-Bohbot, L. Groussin, P. Bouchard, A. Tabarin, P. Chanson, P. Lecomte, I. Guilhem, N. Carrere, E. Mirallié, F. Pattou, J.L. Peix, D. Goere, F. BorsonChazot, P. Caron, V. Bongard, B. Carnaille, P. Goudet, E. Baudin, Long-term results of the surgical management of insulinoma patients with MEN1: a Groupe d'étude des Tumeurs Endocrines (GTE) retrospective study. Eur. J. Endocrinol. 172(3), 309-319 (2015)

18. J.M. De Laat, C.R. Pieterman, M.F. van den Broek, J.W. Twisk, A.R. Hermus, O.M. Dekkers, W.W. de Herder, A.N. van der Horst-Schrivers, M.L. Drent, P.H. Bisschop, B. Havekes, M.R. Vriens, G.D. Valk, Natural course and survival of neuroendocrine tumors of thymus and lung in MEN1 patients. J. Clin. Endocrinol. Metab. 99(9), 3325-3333 (2014)

19. J.M. De Laat, O.M. Dekkers, C.R. Pieterman, W.P. Kluijfhout, A. R. Hermus, A.M. Pereira, A.N. van der Horst-Schrivers, M.L. Drent, P.H. Bisschop, B. Havekes, W.W. de Herder, G.D. Valk, Long-term natural course of pituitary tumors in patients with MEN1: results from the DutchMEN1 study group (DMSG). J. Clin. Endocrinol. Metab. 100(9), 3288-3296 (2015)

20. P. Goudet, C. Bonithon-Kopp, A. Murat, P. Ruszniewski, P. Niccoli, F. Ménégaux, G. Chabrier, F. Borson-Chazot, A. Tabarin, P. Bouchard, G. Cadiot, A. Beckers, I. Guilhem, O. Chabre, P. Caron, H. Du Boullay, B. Verges, C. Cardot-Bauters, Genderrelated differences in MEN1 lesion occurrence and diagnosis: a cohort study of 734 cases from the Groupe d'etude des Tumeurs Endocrines. Eur. J. Endocrinol. 165(1), 97-105 (2011)

21. R.V. Thakker, P.J. Newey, G.V. Walls, J. Bilezikian, H. Dralle, P. R. Ebeling, S. Melmed, A. Sakurai, F. Tonelli, M.L. Brandi, Clinical practice guidelines for multiple endocrine neoplasia type 1 (MEN1). J. Clin. Endocrinol. Metab. 97, 2990-3011 (2012)

22. Rare Diseases Task Force: Patients Registries in the field of rare diseases. http://www.eucerd.eu/?post_type $=$ document $\& p=1218$, accessed December 2016

23. A. Sakurai, S. Suzuki, S. Kosugi, T. Okamoto, S. Uchino, A. Miya, T. Imai, H. Kaji, I. Komoto, D. Miura, M. Yamada, T. Uruno, K. Horiuchi, A. Miyauchi, M. Imamura; MEN Consortium of Japan, T. Fukushima, K. Hanazaki, S. Hirakawa, T. Igarashi, T. Iwatani, M. Kammori, T. Katabami, M. Katai, T. Kikumori, K. Kiribayashi, S. Koizumi, S. Midorikawa, R. Miyabe, T. Munekage, A. Ozawa, K. Shimizu, I. Sugitani, H. Takeyama, M. Yamazaki, Multiple endocrine neoplasia type 1 in Japan: establishment and analysis of a multicentre database. Clin. Endocrinol. 76(4), 533-539 (2012)

24. A. Sakurai, M. Yamazaki, S. Suzuki, T. Fukushima, T. Imai, T. Kikumori, T. Okamoto, K. Horiuchi, S. Uchino, S. Kosugi, M. Yamada, I. Komoto, K. Hanazaki, M. Itoh, T. Kondo, M. Mihara, M. Imamura, Clinical features of insulinoma in patients with multiple endocrine neoplasia type 1: analysis of the database of the MEN Consortium of Japan. Endocr. J. 59(10), 859-866 (2012)

25. C.R. Pieterman, J.M. Schreinemakers, H.P. Koppeschaar, M.R. Vriens, I.H. Rinkes, B.A. Zonnenberg, R.B. van der Luijt, G.D. Valk, Multiple endocrine neoplasia type 1 (MEN1): its manifestations and effect of genetic screening on clinical outcome. Clin. Endocrinol. 70(4), 575-581 (2009)

26. P.J. Newey, R.V. Thakker, Role of multiple endocrine neoplasia type 1 mutational analysis in clinical practice. Endocr. Pract. 17 (3), 8-17 (2011)

27. T.C. Lairmore, L.D. Piersall, M.K. DeBenedetti, W.G. Dilley, M. G. Mutch, A.J. Whelan, B. Zehnbauer, Clinical genetic testing and early surgical intervention in patients with multiple endocrine neoplasia type 1 (MEN 1). Ann. Surg. 239(5), 637-645 discussion 645-647. (2004)

28. J.A. Norton, D.L. Fraker, H.R. Alexander, F. Gibril, D.J. Liewehr, D.J. Venzon, R.T. Jensen, Surgery increases survival in patients with gastrinoma. Ann. Surg. 244(3), 410-419 (2006)

29. R.S. Van Leeuwaarde, B.P. van Nesselrooij, A.R. Hermus, O.M. Dekkers, W.W. de Herder, A.N. van der Horst-Schrivers, M.L. Drent, P.H. Bisschop, B. Havekes, M.R. Vriens, J.M. de Laat, C. R. Pieterman, G.D. Valk, Impact of delay in diagnosis in outcomes in MEN1: results from the Dutch MEN1 study group. J. Clin. Endocrinol. Metab. 101(3), 1159-1165 (2016) 